\title{
Scholarly metrics
}

Stefanie Haustein ${ }^{1}$

1 University of Ottawa

Scholarly metrics are indicators based on recorded events of acts (e.g., viewing, reading, saving, diffusing, mentioning, citing, reusing, modifying) related to scholarly documents (e.g., papers, books, blog posts, datasets, code) or scholarly agents (e.g., researchers, universities, funders, journals). 